Condensed Matter Physics, 1999, Vol. 2, No. 1(17), p. 133-142

\title{
Energy spectrum of transition metal impurity in a semiconductor with an ideal surface
}

\author{
S.V.Melnychuk, I.M.Yurijchuk \\ Chernivtsi State University, \\ 2 Kotsiubynsky Str., 274012 Chernivtsi, Ukraine
}

Received February 19, 1999

Energy spectrum of the transition metal impurity which substitutes a cation in a zink-blende structure semiconductor with an ideal surface is investigated theoretically. Model Hamiltonian of the semiconductor is treated by the semi-empirical tight-binding method, and energy of deep $3 d$-levels is determined by the Green function method within the framework of the Kikoin-Flerov model. Numerical calculations are performed for $\mathrm{Cr}$ impurity atoms in a CdTe semiconductor with an [100] ideal surface. It is shown that in the case when the impurity substitutes an atom near the surface, essential reconstruction of its energy spectrum takes place. One-electron $t_{2}$ - and $e$-levels occurring from $3 d$-volume states for the crystal with an ideal surface due to the lowering of the system symmetry split up. The magnitude of the splitting is rather small, when impurity is situated near but at some distance from the surface, and strongly increases when impurity substitutes an atom directly on an ideal surface. Main mechanisms of oneelectron deep level formation in the forbidden gap of a semiconductor with ideal anion and cation surfaces are determined.

Key words: semiconductor, 3d-impurity, surface, energy spectrum

PACS: 73.20

Semiconductor heterostructures, due to their wide use in modern electronics, have recently aroused much scientific interest [1]. The properties of defects in such systems have not been investigated enough as compared with the case of defects in bulk materials. The main interest was focused on the studies of charged impurities which give shallow levels in the forbidden gap. As to the defects which create deep levels there are to our knowledge only investigations of vacancies and impurities of 6 groups in GaAs/GaAlAs superlattices [2]. The purpose of this paper is to study transition metal impurities in a semiconductor with an ideal surface. Transition elements give deep levels practically in all bulk $\mathrm{A}_{2} \mathrm{~B}_{6}$ semiconductors. Substitution impurities in $\mathrm{A}_{2} \mathrm{~B}_{6}$ semiconductors are neutral, therefore, perturbation induced 
by an impurity is short-range and almost completely defined by the difference of core potentials of the impurity and the matrix. Nowadays the theory of deep levels created by $3 d$-elements in bulk semiconductors has achieved a high level of development [3-5]. It is proved that one-particle states, which form deep levels in the forbidden gap of these semiconductors, emerge due to the resonance scattering of band electrons on unfilled 3d-states $[6,7]$ and not to the potential scattering on the impurity potential as in the case of a usual isoelectron impurity. As a result, deep levels have $d$ - and not $s$ - or $p$-symmetry, while eigen- $d$-levels are repulsed into the forbidden gap and influence the matrix results in a covalent mixing that distorts the atomic function but does not change its point symmetry. The fact that the position of $3 d$-levels in the forbidden gap is defined mainly by the nature of the impurity and not by the semiconductor matrix sets forth an idea to consider impurities as universal levels which are reference points when an interface between two semiconductors is created and give the possibility to define a valence band offset in heterostructure [8]. In paper [9] the opposite approach is proposed: namely, to investigate energy position of $3 d$-levels in a heterostructure in comparison with their position in initial bulk semiconductors. In $[9,10]$ the theory of deep levels created by transition metal impurities in semiconductor superlattices is developed. This approach is based on a theoretical model which was successfully used for the investigation of chemical trends in $\mathrm{A}_{2} \mathrm{~B}_{6}$ semiconductors and appears to be very clear for understanding the $3 d$-levels nature [3]. As it was mentioned above, the main features of the impurity behaviour are determined by $3 d$-orbitals and not by the matrix. But there are specific situations when the matrix essentially influences the energy spectrum of the impurity. As it will be shown further, this is when the impurity changes its position in the crystal towards the interface. In this paper we consider the case of a sharp interface of a semiconductor with vacuum, i.e. an ideal surface. Fundamental peculiarities of the behaviour of $3 d$-levels on an ideal surface should also be present in the case of other hererostructures. A crystal with an ideal surface can be treated as a semi-infinite set of crystallographic planes parallel to the plane which forms an interface of the crystal with vacuum. For a zink-blende semiconductor with an ideal surface perpendicular to the [100] direction, each atomic plane comprises only identical atoms (cations or anions) and is sandwiched between unlike atomic planes. We chose a coordinate system such that the OZ axis is parallel to the [100] direction and axes OX, OY are directed along the basis vectors $\boldsymbol{a}_{1}, \boldsymbol{a}_{2}$ of a two-dimensional lattice. The equilibrium position of the atom in the superlattice is defined by the vector

$$
R\left(n N_{p} l k\right)=l_{1} \boldsymbol{a}_{1}+l_{2} \boldsymbol{a}_{2}+\boldsymbol{\tau}_{\perp}^{p k}+\boldsymbol{\tau}_{\|}^{p k} .
$$

Here $N_{p}$ is the number of the atomic plane $1 \leqslant N_{p} \leqslant \infty ; l_{1}, l_{2}$ are integers; vector $\tau_{\perp}^{p k}$ specifies the position of the atomic plane $N_{p}$ and the position of $k$-th atom in the atomic plane $N_{p}$ inside an elementary two-dimensional cell is given by $\boldsymbol{\tau}_{\|}^{p k}$ $(k=1,2)$.

The characteristic feature of impurities with a semi-filled $3 \mathrm{~d}$-shell consist in the fact that the impurity potential leads to the appearance of $3 d$-levels in the 
forbidden gap or in its vicinity. Therefore, as the basis for the system we choose $[6]$ :

$$
\Phi_{i}=\left\{\Psi_{k a}, \Psi_{d \mu}\right\},
$$

where $\Psi_{k a}$ is a wave function of band electrons and $\Psi_{d \mu}$-one of $d$-electrons; $\boldsymbol{k}$ is a vector in the Brillouin zone.

We suggest that the impurity substitute an atom in a cation atomic plane of the crystal with an ideal surface. The impurity has four nearest neighbour anion atoms from the adjacent planes. One-electron Schrodinger equation for a semiconductor with an impurity is given by [6]

$$
\left[-\frac{\hbar^{2} \nabla^{2}}{2 m}+\sum_{j \neq 0} U_{h}\left(\boldsymbol{r}-\boldsymbol{R}_{j}\right)+U_{s}\left(\boldsymbol{r}-\boldsymbol{R}_{0}\right)-E\right] \Psi_{i}(\boldsymbol{r})=0,
$$

where $\sum_{j \neq 0} U_{h}\left(\boldsymbol{r}-\boldsymbol{R}_{j}\right)$ is a ligand crystal field, $U_{s}\left(\boldsymbol{r}-\boldsymbol{R}_{0}\right)$ is a substitution impurity potential

$$
U_{s}\left(\boldsymbol{r}-\boldsymbol{R}_{0}\right)=U_{d}\left(\boldsymbol{r}-\boldsymbol{R}_{0}\right)-U_{h}\left(\boldsymbol{r}-\boldsymbol{R}_{0}\right)+U_{1}\{\Delta \varrho(\boldsymbol{r})\} .
$$

Here $U_{d}, U_{h}$ are core potentials of the impurity and the ion, $U_{1}\{\Delta \varrho(\boldsymbol{r})\}$ is a change of the valency electron potential induced by a redistribution of the electron density; the self-consistent potential $U_{d}\left(\boldsymbol{r}-\boldsymbol{R}_{0}\right)$ for $d$-electrons enters the Schrodinger equation for eigen- $d$-functions $\psi_{d m}$

$$
\left[-\frac{\hbar^{2} \nabla^{2}}{2 m}+U_{d}\left(\boldsymbol{r}-\boldsymbol{R}_{0}\right)-\varepsilon_{d}\right] \psi_{d m}\left(\boldsymbol{r}-\boldsymbol{R}_{0}\right)=0
$$

where $m$ is a projection of the orbital moment $l=2$. The solution of equation (2) can be presented as an expansion on the basis (1)

$$
\Psi_{i \gamma \mu}(\boldsymbol{r})=A_{\gamma}^{-1 / 2}\left[\Psi_{d \gamma \mu}(\boldsymbol{r})+\Psi_{b \gamma \mu}(\boldsymbol{r})\right] .
$$

In (3), $\mu$ numbers the rows of irreducible representation $\gamma, A_{\mu}$ is a scale factor; $\Psi_{d \gamma \mu}(\boldsymbol{r})$ are functions localized near the impurity centre and are a linear combination of the atomic orbitals $\phi_{m}(\boldsymbol{r})$

$$
\Psi_{d \gamma \mu}(\boldsymbol{r})=F_{d}^{\gamma \mu} \sum_{m} u_{m}^{\gamma \mu} \phi_{m}(\boldsymbol{r})=F_{d}^{\gamma \mu} \varphi_{\gamma \mu}(\boldsymbol{r})
$$

and

$$
\Psi_{d \gamma \mu}(\boldsymbol{r})=\sum_{\boldsymbol{k} a} F_{\boldsymbol{k} a}^{\gamma \mu} \tilde{\varphi}_{\boldsymbol{k} a}\left(\boldsymbol{r}, \boldsymbol{R}_{0}\right)
$$

is a linear superposition of Bloch states of a pure crystal.

Inserting (3) in (2), taking into account (4) and (5) and considering the approximation when the contribution from the potential scattering can be omitted as compared with the contribution from resonance scattering we obtain an equation for deep energy levels:

$$
E_{i \gamma}-\varepsilon_{\gamma}=M_{\gamma}(E)
$$


where

$$
\begin{gathered}
\varepsilon_{\gamma}=\varepsilon_{d}+\Delta \varepsilon_{d}+\langle\gamma \mu|W| \gamma \mu\rangle, \\
M_{\gamma}(E)=\sum_{\boldsymbol{k} a} \frac{\langle\gamma \mu|W| \boldsymbol{k} a\rangle\langle\boldsymbol{k} a|W| \gamma \mu\rangle}{E-\varepsilon_{\boldsymbol{k} a}}=\left\langle\gamma \mu\left|W G_{0}(E)\right| \gamma \mu\right\rangle .
\end{gathered}
$$

Here $W$ is a crystal field, e.g., the total potential of matrix ions and valency electrons, which splits $d$-states into $t_{2^{-}}$and $e$-levels; $G_{0}(E)$ is a Green function of a pure crystal with an ideal surface; $\varepsilon_{\boldsymbol{k} a}$ is a band spectrum of the crystal. For the numerical analysis we use a semi-empirical tight-binding model $[12,13]$ which is comparatively simple in practical implementation but gives a clear and comprehensive picture of chemical tendencies in the system. In the tight-binding method a wave function of the crystal is presented as a linear combination of atomic orbitals centred in different atoms. For $\mathrm{A}_{2} \mathrm{~B}_{6}$ semiconductors it is sufficient to include in the basis $s$ - and $p$-orbitals and to take into account an interaction with the nearest neighbours only. Thus, a one-electron wave function of the crystal is given as a linear combination of $s$ - and $p$ - tight-binding orbitals centred on sites $R\left(n N_{p} l k\right)$

$$
\phi_{n \boldsymbol{k} a}(\boldsymbol{r})=\sum_{i N_{p}} C_{i N_{p}}(n \boldsymbol{k} a) \varphi_{i N_{p}}(\boldsymbol{r}) .
$$

In the basis (9), we obtain a secular matrix for the energy band spectrum of the crystal with the surface

$$
\left|H_{i N_{p}, j N_{p}}^{(0)}\left(n, \boldsymbol{k}_{\|}+\boldsymbol{k}_{\perp}, a\right)-E \delta_{i j}\right|=0 .
$$

The Hamiltonian matrix $H_{i N_{p}, j N_{p}}^{(0)}\left(n, \boldsymbol{k}_{\|}+\boldsymbol{k}_{\perp}, a\right)$ is constructed with the use of a set of parameters deduced from the available experimental data of bulk materials [13].

Projection of the Green functions $G_{0}(E)$ on the tight-binding orbitals has the form:

$$
G_{i N_{p}, j N_{p}}^{(0)}(E)=\frac{1}{N} \sum_{\boldsymbol{k} a} \frac{C_{j N_{p}^{\prime}}(n \boldsymbol{k} a) C_{i N_{p}}^{*}(n \boldsymbol{k} a)}{\varepsilon-\varepsilon_{n \boldsymbol{k} a}} .
$$

For the calculation of matrix elements $\langle\boldsymbol{k} a|W| \gamma \mu\rangle$ in (8), the following relation was used [14]:

$$
\langle\boldsymbol{k} a|W| \gamma \mu\rangle=\left\langle\boldsymbol{k} a\left|V_{d}-V_{h}\right| \gamma \mu\right\rangle+\left(\varepsilon_{\boldsymbol{k} a}-\varepsilon_{d}\right)\langle\boldsymbol{k} a \mid \gamma \mu\rangle .
$$

Here $U_{s}=\left(V_{d}-V_{h}\right)$ is an impurity potential which we treat in the Hartree-FockSlater approximation:

$$
\begin{aligned}
& U_{s}(\boldsymbol{r})=V_{d}^{2+}\left(\boldsymbol{r}-\boldsymbol{R}_{0}\right)-V_{h}^{2+}\left(\boldsymbol{r}-\boldsymbol{R}_{0}\right) ; \\
& V(\boldsymbol{r})=\frac{2 Z_{0}}{r}-\frac{2}{r} \int_{0}^{r} \sigma(t) \mathrm{d} t-6\left(\frac{3}{8} \varrho(r)\right)^{1 / 3},
\end{aligned}
$$

where $Z_{0}$ is an atomic number, $\varrho(r)=\left(4 \pi r^{2}\right)^{-1} \sigma(r)$ is the averaged electron density. With the obtained potential two-centre integrals entering (12) were defined. We 
choose atomic functions [15] as eigen-functions of 3d-electrons. Equation (6) for the evaluation of deep level energies was solved numerically. Energy dependencies $M_{\gamma}(E)$ were defined from relation (8) where eigenvalues $\varepsilon_{\boldsymbol{k} a}$ and the eigenfunctions of a pure crystal entering $G^{(0)}(E)$ were obtained by diagonalizing the Hamiltonian matrix (10). Intersections of $E-\varepsilon_{\gamma}$ with $M_{\gamma}(E)$ give one-electron energy of $3 d-$ levels of transition metal impurities in the forbidden gap of semiconductors.

$\mathrm{E}, \mathrm{eV}$

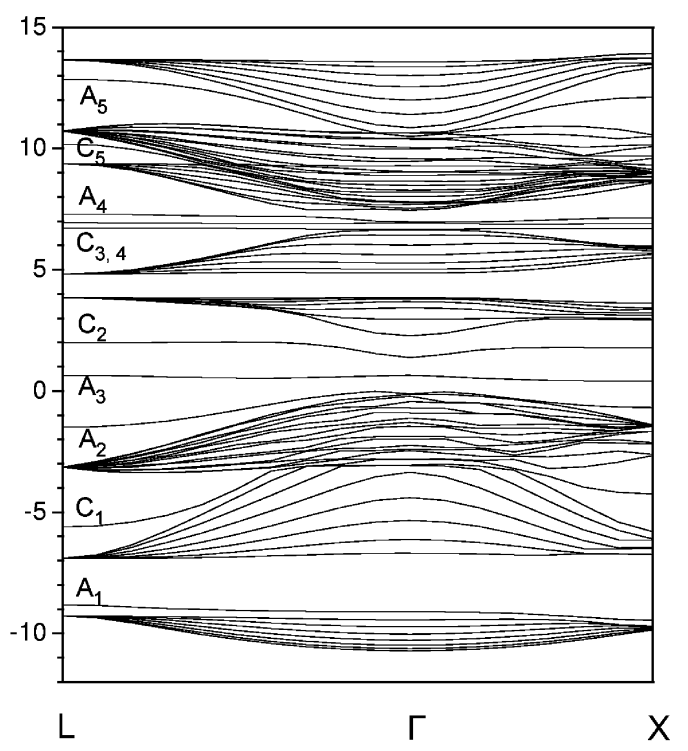

Figure 1. Band structure of semiconductor CdTe [100] ideal surface. Bound surface states are denoted by $C_{i}$ (for cation surface) and $A_{i}$ (for anion surface). A set of tight-binding parameters used for the band structure calculations is given in [13].

Relations (6)-(8) are similar to those of bulk materials $[6,7]$. But there are essential differences. An expression for $M_{\gamma}(E)$ contains a Green function whose projection on the local basis depends on index $N_{p}$. Thus, the energy of deep levels $E_{i \gamma}$ also depends on this index, e.g., on whose atomic plane $N_{p}$ an impurity substitutes a host atom. Furthermore, the presence of an ideal surface lowers the system symmetry. In the case of an [100] ideal surface, the point symmetry of a zink-blende crystal lowers from $\mathrm{T}_{d}$ to $\mathrm{C}_{2 v}$. Degenerated $t_{2}$ - and $e$-levels split up in the $\mathrm{C}_{2 v}$ crystal field to $t_{2}=a_{1}+b_{1}+b_{2}, e=a_{1}+a_{2}$. Therefore, index $\gamma$ in (6)-(8) enumerates irreducible representation of the $\mathrm{C}_{2 v}$ group: $a_{1}, a_{2}, b_{1}, b_{2}$.

Generally speaking, the Hamiltonian matrix of a crystal with an ideal surface is of an infinite dimension. Therefore, in numerical calculations for surface modelling a slab method is used $[10,11]$, in which a semi-infinite crystal is confined by the second surface identical to the first. The system is modelled by a slab with a finite width but infinite in the direction parallel to the surface. For the surface band structure calculation typically 12-15 layers are enough to be taken into consideration. The slab of this width simulates the bulk, as well as the surface band structure. Wave functions on the opposite surfaces do not intersect with each other and the energy spectrum practically does not change when more layers are added to the slab.

Let us consider in more detail the peculiarities of the formation of an [100] ideal surface in a zink-blende crystal. When a surface is formed, the surface crystal bonds are broken and an atom on the surface lacks one or more neighbours. Each atom in an [100] ideal surface has two bulk and two broken bonds. Interaction between neighbour atoms and the existence of a translation symmetry parallel to the surface result in the splitting of dehybridized states into the bands of surface 
states. These states are localized near the surface when their energies lie in the gaps of the energy spectrum of the crystal (bound surface states).

The calculated band structure of a [100] surface along some high symmetry directions of the Brillouin zone for the semiconductor crystal CdTe is presented in figure 1 . The [100] surface is polar, therefore it is necessary to distinguish two cases depending on whether the surface consists of cations $(\mathrm{Cd}[100])$ or anions (Te[100]), respectively. Bound surface states for the both ideal surfaces are presented in the same figure and denoted as $\mathrm{C}_{i}$ and $A_{i}$, respectively. As it follows from figure 1 , as the result of surface creation, groups of surface states emerge on the background of the bulk energy spectrum. In the case of an anion surface there are five surface bands. $A_{1}$-states split up from sorbitals, and $A_{2}, A_{3}$ - from p-orbitals of the valency band. In the case of a cation surface there are also five surface bands. $C_{1^{-}}, C_{2^{-}}$states split up from cation s-orbitals.

Analyzing partial densities of electron states projected on each atomic

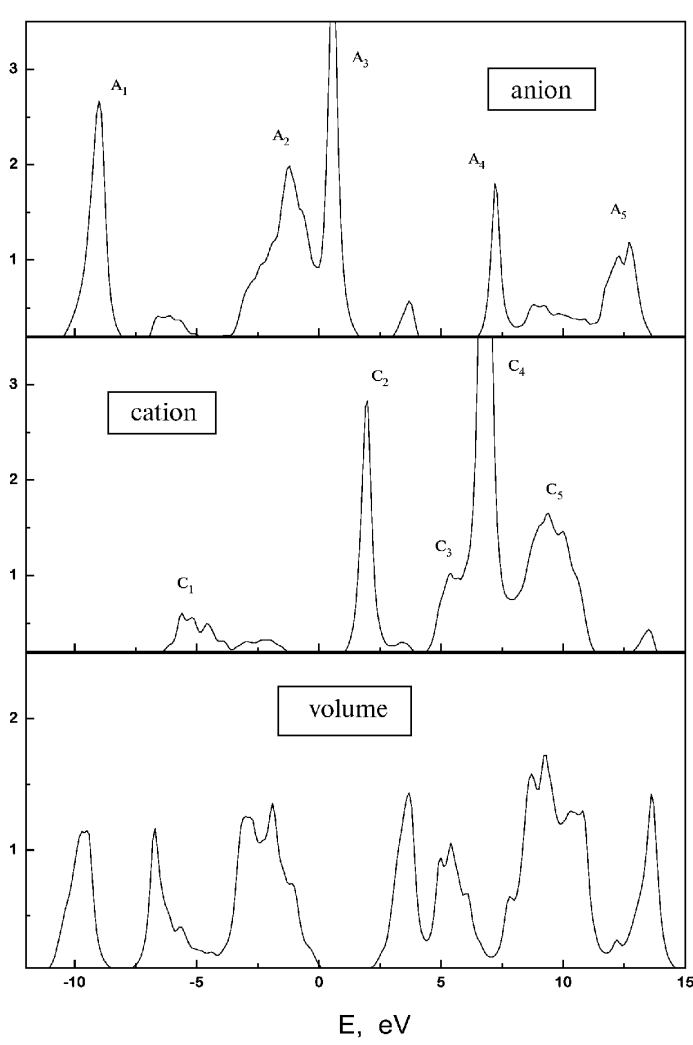

Figure 2. Densities of electron states of semiconductor CdTe projected on ideal anion and cation surfaces. plane (figure 2), the origin of surface states and their localization have been found out. Projected densities were obtained as an imaginary part of Green functions (11) at $N_{p}=N_{p}^{\prime}$. As follows from figure 2, peaks responsible for surface bands are absent in the bulk density, hence these states are almost completely localized on the surfaces which form an ideal surface. Here it is important to note the presence of three bands situated in the forbidden gap: $C_{2}$ are states which lie slightly below the conduction band, and $A_{2}$ and $A_{3}$ are states which lie near the valency band edge.

The energy of deep levels caused by $\mathrm{Cr}$ impurity in CdTe is found as the solution of equation (6). Calculating matrix elements (8), we assume that the impurity potential does not change essentially compared with that of the bulk material. In figures 3,4 we present the results of the solution of equation (6) in the case when the impurity is situated near the cation (figure 3) or the anion (figure 4) ideal surface. Index $N_{p}$ numbers atomic planes in an ascending order of the distance from the surface. $N_{p}=1$ denotes an atomic plane which forms an ideal surface: for an anion surface $3 d$-impurity substitutes an atom in atomic planes with odd $N_{p}$, and for a cation one - with even $N_{p}$. Values $M_{\gamma}(E)$ calculated for an impurity 
substituting an atom far enough from the surface are represented in figures 3,4 by bold curves. It should be noted that the results for the impurity located in the volume of the crystal $\left(N_{p}>12\right)$ coincide with the calculations for bulk CdTe [7]. The values $M_{\gamma}(E)$ for different irreducible representations $\gamma$, when approaching the surface (from $N_{p}=7,8$ ), differ among themselves. It means that accidentally degenerate, $\left(t_{2}\right.$-threefold, $e$-twofold) deep levels split up and their energy positions move. In figure 5 we present the energies of deep levels for impurity $\mathrm{Cr}$ in $\mathrm{CdTe}$ depending on the number of atomic plane $N_{p}$.

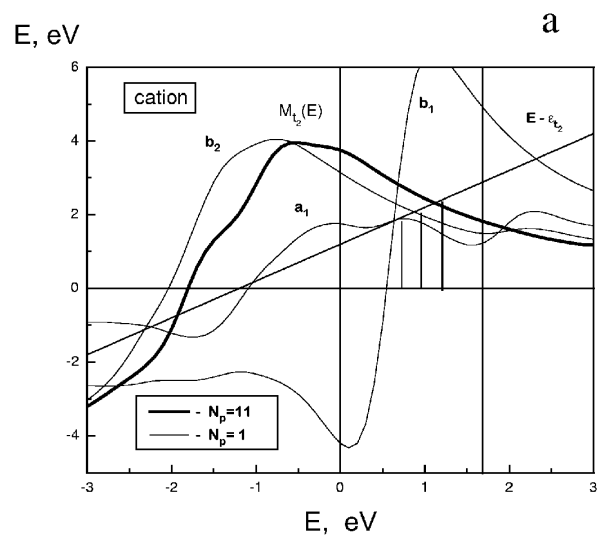

$E, e V$

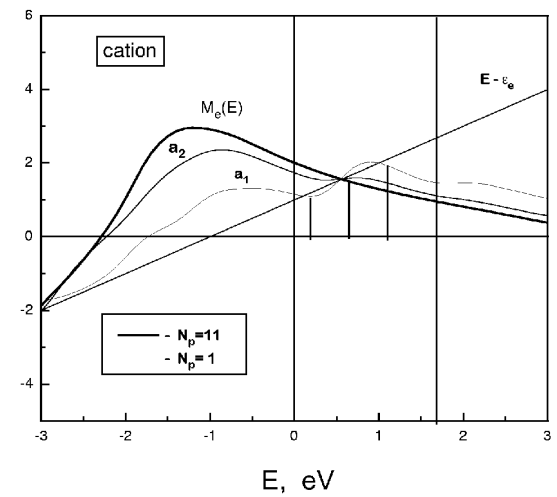

Figure 3. Graphic solution of equation (9) for the system CdTe:Cr in the case of a cation ideal surface: a - t2level, b - e-level. Bold curves denoted by $\mathrm{M}(\mathrm{E})$ are calculated for an impurity substituting an atom in the volume of the crystal.

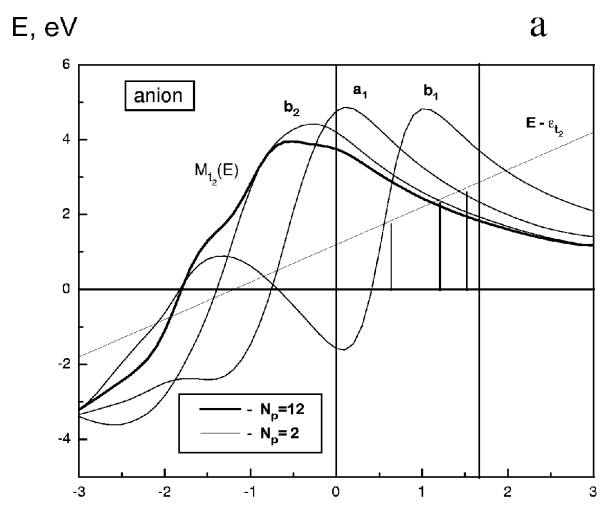

$E$, eV

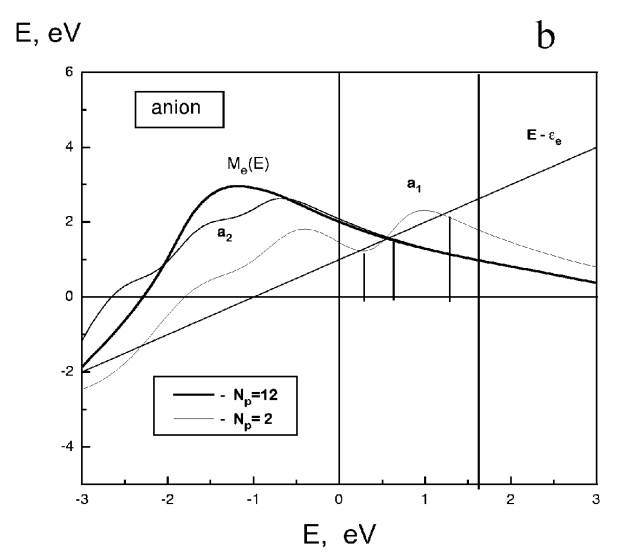

Figure 4. Graphic solution of equation (9) for the system CdTe:Cr in the case of an anion ideal surface: a - t2level, b - e-level. Bold curves denoted by $\mathrm{M}(\mathrm{E})$ are calculated for an impurity substituting an atom in the volume of the crystal.

Analyzing figures 3,4 several features common for two different surfaces should be noted. A cation surface, as well as an anion surface influence $t_{2}$-states stronger than $e$-states. For the both surfaces $a_{2}$-states, which split up from volume $e$-states 
when approaching the surface, practically do not change their energy position, and $a_{1}$-states give two additional levels situated at almost equal distances from the original state. The influence of the surface on renormalization of $M_{t 2}(E)$ is much stronger and is especially significant for a cation surface (figure 5a). It is evident, since in this case the impurity is situated directly on an ideal surface. Energy dependencies $M_{\gamma}(E)$ for $\gamma=b_{1}$ splitting up from volume $t_{2}$-states are practically the same (they change the sign in the forbidden gap), whereas $M_{\gamma}(E)$ for $\gamma=a_{1}, b_{2}$ differ for two different surfaces: they move to lower energies for a cation surface and vice versa for an anion surface. $A_{1^{-}}, b_{2}$-levels move in different directions from the volume level depending on the type of the surface. Such behaviour is caused by two surface bands which are situated near the valency band edge $\left(A_{2}, A_{3}\right.$ in figure 1$)$. These bands occur as the result of dehybridization of anion bonds and are located slightly above the hybridized p-states responsible for valency band mixing. Besides, as can be shown from

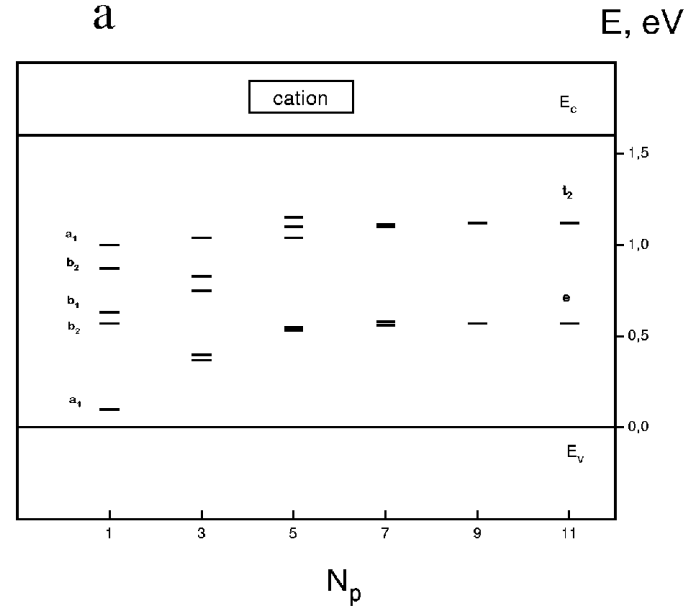

b $\mathrm{E}, \mathrm{eV}$

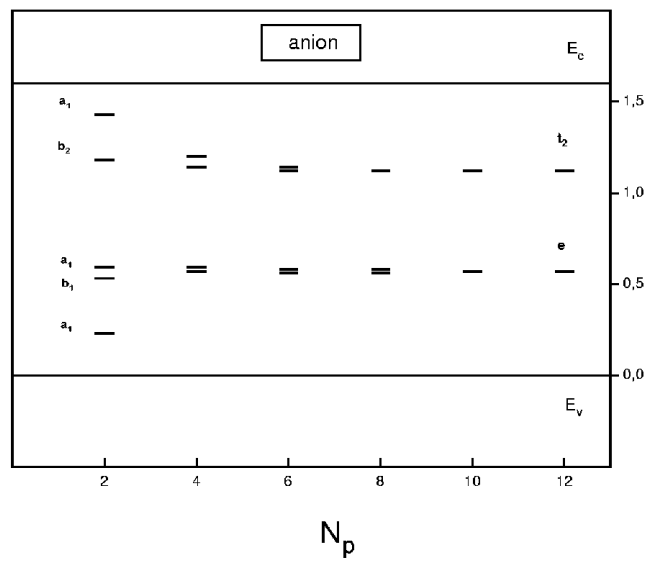

Figure 5. Deep level energies of the system CdTe:Cr depending on the distance from a-cation and b-anion ideal surfaces. figure 2, these bands give the greatest contribution to the projected densities of states. It results in a shift of deep $t_{2}$-levels to high energies: the mixing of $t_{2}$-levels with surface bands adds to the mechanism of the covalent mixing of $t_{2}$-levels with valency electrons, the latter is responsible for the formation of $3 d$-deep levels in the volume of the crystal. The density of anion surface states for a cation surface is rather small and, therefore, the respective hybridization is negligible.

Numerical calculations of deep level energies of $\mathrm{Cr}$ impurity in CdTe, when the impurity substitutes an atom near an [100] ideal surface and in the volume of the crystal allow us to draw such conclusions. Deep $t_{2^{-}}$and $e$-levels occurring from $3 d$-volume states for a crystal with an ideal surface due to the lowering of the system symmetry split up. The magnitude of splitting is rather small, when the impurity is situated near but at some distance (about 3-5 atomic planes) from the surface, and strongly increases when the impurity substitutes an atom directly on an ideal surface (up to $1 \mathrm{eV}$ ). The second important conclusion is the possibility 
of the situation when the levels occurring from $t_{2}$-states as the result of splitting and the energy shift appear to be situated below the levels occurring from $e$-states. Thus, an inversion of deep levels as compared with the volume crystal is possible.

An ideal surface [100] of zink-blende semiconductors is polar. Therefore, there are two types of surfaces which influence the energy spectrum of $3 d$-impurity in different ways. Reconstruction of the impurity energy spectrum is much stronger for states occurring from $t_{2}$-levels and also when the impurity is situated directly on an ideal surface (the case of a cation surface). Function $M_{\gamma}(E)$ reveals in these cases a sharper behaviour and, accordingly, the amount of splitting increases. The main feature of $t_{2}$-level behaviour near an anion ideal surface is its splitting and the energy shift to high energies in the forbidden gap (even the repulsion of levels to the conduction band). For a cation surface the inverse situation takes place. These differences are due to the existence on an anion ideal surface of the bands emerging as the result of dehybridization of valency band p-states. The covalent mixing of $3 d-$ states with anion surface bands is the main mechanism of the creation of deep levels in the forbidden gap in a semiconductor with an ideal anion surface. One-electron deep-levels calculated in the present paper are the basis for the construction of multiplet terms of transition metal impurities in the forbidden gap of a crystal with an ideal surface and the determination of their ionization energies.

\section{References}

1. Herman R.E. Semiconductor Superlattices, Moscow, Mir, 1989 (in Russian).

2. Ren Y.R., Dow J.D., Shen J. Deep impurity levels in semiconductors superlattices. // Phys. Rev. B., 1988, vol. 38, No. 15, p. 10677-10692.

3. Kikoin K.A. Electronic Properties of Transition Metal Impurities in Semiconductors. Moscow, Energoatomizdat, 1991 (in Russian).

4. Sokolov V.I., Kikoin K.A. Exitons bound to impurities of 3d elements in II-VI compounds. Soviet Scientific Reviews. Section A., vol. 12, 1989 (in Russian).

5. Zunger A. Electronic structure of 3d transition-atom impurities in semiconductors. // Solid Stat. Phys., Orland, Acad. Press., 1986, vol. 39, p. 276-464.

6. Kikoin K.A., Fleurov V.N. // J. Phys. C., 1984, vol. 17, p. 2357-2362.

7. Kikoin K.A., Kurek I.G., Melnychuk S.V. Energy spectrum of 3d-impurities in CdTe. // FTS, 1989, vol. 23, No. 12, p. 2153-2158 (in Russian).

8. Langer J.M., Delerue C., Lannoo M., Heinrich H. Transition-metal impurities in semiconductors and heterojunction lineups. // Phys. Rev. B., 1988, vol. 38, No. 11, p. 77237739 .

9. Kikoin K.A., Manakova L.A. Impurity states of 3d-ions in I-type superlattices. Preprint of the Russian scientific centre "Kurchatovski Institut", N 5677/9, 1993, 15 p. (in Russian).

10. Kikoin K.A., Manakova L.A. Impurity states of 3d-ions in quantum pits and I-type superlattices. // FTS, 1995, vol. 29, No. 2, p. 291-305 (in Russian).

11. Bechshtedt F., Enderlain R. Semiconductors Surfaces and Interfaces, Moscow, Mir, 1990 (in Russian). 
12. Harrison W. Electronic Structure and Properties of Solid States, Moscow, Mir, 1983 (in Russian).

13. Vogl P., Hjalmarson H.P., Dow J.D. A semiempirical tightbinding theory of the electronic structure of semicoductors. // J. Phys. Chem. Sol., 1983, vol. 44, No. 5, p. 365378.

14. Kikoin K.A., Kurek I.G., Melnichuk S.V. Crystal fields and ligand fields for 3dimpurities in CdTe. Preprint of I.V.Kurchatov Institute of Atomic energy, N 6016/9, 1990, 21 p.

15. Froese C. Hartree-Fock results, He to Rn. // Atomic Data, 1972, vol. 4, p. 301-399.

\title{
Спектр домішки перехідного металу в напівпровіднику з ідеальною поверхнею
}

\author{
С.В.Мельничук, І.М.Юрійчук \\ Чернівецький державний університет, \\ 274012 Чернівці, вул. Коцюбинського, 6 \\ Отримано 19 лютого 1999 р.
}

Теоретично досліджується енергетичний спектр домішки з незаповненою $3 d$-оболонкою, що заміщує катіон в напівпровіднику з структурою цинкової обманки у випадку наявності в кристалі ідеальної поверхні. Модельний гамільтоніан напівпровідника будувався в моделі напівемпіричного сильного зв'язку, а енергії глибоких $3 d$-рівнів визначалися методом функцій Гріна в рамках моделі Кікоєна-Флерова. Числові розрахунки виконані для домішки $\mathrm{Cr}$ в напівпровіднику $\mathrm{CdTe}$ з ідеальною поверхнею [100]. Показано, що у випадку розташування домішки поблизу поверхні відбувається суттєва перебудова їі енергетичного спектру. Одноелектронні $t_{2}$ - і $e$-рівні, що обумовлені $3 d-$ центрами в об'ємному напівпровіднику для системи, обмеженої ідеальною поверхнею, внаслідок пониження симетрії розщеплюються. Величина розщеплення різко зростає для домішки, розташованої безпосередньо на атомній площині, що утворює ідеальну поверхню. Внаслідок полярності поверхні [100] напівпровідника CdTe можливе існування двох типів поверхні, які по різному впливають на енергетичний спектр $3 d$-домішок. Встановлено основні механізми формування одноелектронних глибоких рівнів в забороненій зоні напівпровідника для двох типів поверхні.

Ключові слова: напівпровідник, 3d-домішка, поверхня, енергетичний спектр

PACS: 73.20 